OPEN

SUBJECT AREAS:

STEM-CELL

DIFFERENTIATION

MESENCHYMAL STEM CELLS

Received

6 January 2014

Accepted

10 March 2014

Published

8 April 2014

Correspondence and requests for materials should be addressed to T.H. (toshiohamatani@ z3.keio.jp)

\section{Derivation of human decidua-like cells from amnion and menstrual blood}

\author{
Kana Sugawara ', , Toshio Hamatani' , Mitsutoshi Yamada', Seiji Ogawa' , Shintaro Kamijo' , Naoaki Kuji', \\ Hidenori Akutsu², Kenji Miyado ${ }^{2}$, Yasunori Yoshimura' \& Akihiro Umezawa²
}

'Department of Obstetrics and Gynecology, Keio University School of Medicine, 35 Shinanomachi Shinjuku-ku, Tokyo 160-8582, Japan, ${ }^{2}$ Department of Reproductive Biology, National Research Institute for Child Health and Development, 2-10-1 Ohkura Setagaya-ku, Tokyo 157-8535, Japan.

We induced differentiation of human amnion-derived mesenchymal stem cells (AMCs) and menstrual blood-derived mesenchymal stem cells (MMCs) into endometrial stroma-like cells, which could be useful for cell therapy to support embryo implantation. Interestingly, the expression patterns of surface markers were similar among AMCs, MMCs, and endometrial stromal cells. In addition, whereas treatment with estrogen and progesterone was not very effective for decidualizing AMCs and MMCs, treatment with 8-Br-cAMP prompted remarkable morphological changes in these cells as well as increased expression of decidualization markers (prolactin and insulin-like growth factor binding protein-1) and attenuated expression of surface markers unique to mesenchymal stem cells. These results demonstrated that bone marrow-derived stem cells, which are considered a potential source of endometrial progenitor cells, as well as AMCs and MMCs show in vitro decidualization potential, which is characteristic of endometrial stromal cells.

M any infertile couples have achieved pregnancy by assisted reproductive technology (ART). However, despite having good-quality embryos for transfer, there are still women who experience repeated implantation failure even after several ART attempts ${ }^{1,2}$. Uterine receptivity is considered as another important factor for successful implantation and many studies have attempted to identify clinically useful markers of a receptive uterine state. However, such molecular markers related to repeated implantation failure have proven difficult to find, largely because uterine receptivity is regulated via the expression of various mediators, including cell adhesion molecules (e.g. cadherins and integrins), chemical mediators (e.g. prostaglandins), and cytokines (e.g. leukemia inhibitory factor and epithelial growth factor).

Thinning of endometrium has also been correlated with implantation failure, with causes including aging ${ }^{3}$, and repeated invasive procedures such as dilation and curettage for miscarriage or early-stage endometrial cancer. Some studies have reported clinical benefits from ascorbic acid (vitamin C), tocopherol (vitamin E), pentoxifylline (PTX), or sildenafil for repeated implantation failure in patients with thin endometrium, although the effectiveness and molecular mechanisms by which such agent could improve the implantation process is not well established ${ }^{4-6}$.

In studies to improve implantation rates, Landgren et al. ${ }^{7}$ developed a coculture model of embryos with endometrial cells harvested from an endometrial biopsy taken at 4, 5, and 6 days after the lutenizing hormone (LH) peak in healthy women with normal menstrual cycles. The rate of pregnancy increased for embryos transferred after the coculture of embryo with endometrium than for embryos transferred after repeat ART ${ }^{8}$. However it is difficult to retrieve and culture endometrial cells from women whose endometrium has already become thin. Therefore we focused on mesenchymal stem cells (MSCs) as a source for this type of cell therapy using endometrial stroma-like cells. MSCs might also support embryo implantation by excreting cytokines and chemical mediators for adhesion, migration, or immunomodulation ${ }^{9-11}$. Indeed, fertility was restored in a patient with severe Asherman's syndrome treated using autologous bone marrow-derived MSC populations ${ }^{12}$.

MSCs are multipotent, adherent stem cells capable of differentiating into osteoblasts, adipocytes, and chondroblasts $^{13}$, as well as endodermal lineages such as pancreatic islands ${ }^{14,15}$ or hepatocytes ${ }^{16,17}$, and ectodermal lineage such as neurons ${ }^{14,18}$. An earlier study verified that human bone marrow-derived MSC (BMCs) are potential progenitors of endometrial stromal cells by demonstrating their ability to decidualize ${ }^{19}$. Decidualization is a remodeling process designed to prepare endometrium for pregnancy that is induced by progesterone secreted from the ovaries in the luteal phase. This remodeling is necessary in a successful pregnancy to regulate trophoblast invasion, resist oxidative stress, and protect the placental semi-allograft against maternal 
immune responses. Endometrial stromal cells (ESCs) are reprogrammed into decidual cells, and display characteristic morphological changes from an elongated spindle-shaped fibroblastic appearance to a polygonal shape with large nuclei. Prolactin (PRL) and insulin-like growth factor binding protein-1 (IGFBP1) have been widely used as phenotypic markers of decidual cells. In the current study, we hypothesized that if MSCs could differentiate into ESC-like cells, their treatment with agents known to promote decidualization could induce the expression of PRL and IGFBP1 in the MSC-derived cells. Indeed, AMCs and MMCs successfully differentiate into endometrial stroma-like cells presenting decidualization potential to the same extent as bone marrow-derived cells ${ }^{19}$. In this context, human amnion in particular could be a powerful therapeutic cell source because not only is it an easily accessible tissue for cell harvesting, it does not require co-administration of immunosuppressive agent or matching of MHC typing for immunological tolerance.

\section{Results}

Characterization of human endometrial cells. Immunofluorescence staining and flow cytometric analysis of cultured human endometrial cells and MRC5 fibroblasts revealed both to be positive for vimentin and negative for cytokeratin, whereas a human carcinoma cell line, HeLa cells, was positive for both vimentin and cytokeratin. (Fig. 1AE). By flow cytometry, the endometrial cells and fibroblasts were negative for CD9 and positive for CD10, whereas HeLa cells were positive for CD9 and negative for CD10. Together, these results confirmed that the endometrial cells were stromal in origin, compared to the epithelial-derived HeLa cells.

We then investigated cell surface marker expressions of ESCs, AMCs, MMCs, BMCs, and MRC5 by flow cytometric analysis on the initial day of treatment (day 0) (Fig. 1F). Although there is no marker specific to MSCs, they are known to express CD73, CD90, and $\mathrm{CD} 105^{20}$, and the AMCs and MMCs tested herein indeed expressed these cell surface markers as well as CD54, CD166 and HLA-ABC, but not HLA-DR (Supplementary Fig. S1). This result confirmed the earlier studies of our group demonstrating that both AMCs and MMCs express MSC markers such as CD29, CD44, CD59, CD73, CD105, and CD166, but not hematopoietic markers such as CD14, CD34, and CD $45^{21-23}$. None of the cells analyzed in the present study expressed hematopoietic lineage markers such as CD34 or monocyte-macrophage antigens such as CD14 (a marker for macrophage and dendritic cells) and CD45 (leukocyte common antigen), suggesting no contaminating hematopoietic cells. Lastly, and interestingly, the surface marker expression patterns of AMCs and MMCs were very similar to those of ESCs.

Decidual differentiation by treatment with $\mathbf{E}_{2}+\mathbf{P}_{4}$. Human ESCs showed distinct morphological changes following treatment with $\mathrm{E}_{2}$ $+\mathrm{P}_{4}$, from spindle-shaped to large round cells with large round nuclei, whereas AMCs, MMCs, and BMCs showed no remarkable morphological changes (Fig. 2A). Flow cytometric analysis of ESCs, AMCs, MMCs, BMCs, and MRC5 fibroblasts on day 14 of treatment with $\mathrm{E}_{2}$ $+\mathrm{P}_{4}$ (Fig. $2 \mathrm{~B}$ and Supplementary Fig. S2) also showed no remarkable changes in surface marker expressions compared to those on day 0 .

Immunofluorescence staining was also performed to assess the expression of decidualization markers (PRL and IGFBP1) at day 0 , 7, and 14 after treatment with $\mathrm{E}_{2}+\mathrm{P}_{4}$ (Fig. 3). None of these markers were expressed at day 0 in ESCs, AMCs, MMCs, and BMCs, but PRL and IGFBP1 were expressed in ESCs on days 7 and 14 following treatment with $\mathrm{E}_{2}+\mathrm{P}_{4}$ (Figs. $3 \mathrm{~A}$ and $3 \mathrm{~B}$ ). AMCs also showed clear expression of PRL on days 7 and 14 following treatment, but only faint IGFBP1 expression (Figs. 3C and 3D), while the reverse pattern was observed in MMCs (Figs. 3E and 3F). Finally, both PRL and IGFBP1 were faintly expressed in BMCs (Figs. $3 \mathrm{G}$ and $3 \mathrm{H}$ ).

The qRT-PCR analysis of expression changes in PRL and IGFBPI mRNAs with $\mathrm{E}_{2}+\mathrm{P}_{4}$ treatment (Figs. $2 \mathrm{C}$ and $2 \mathrm{D}$ ) revealed upregulation of $P R L$ in each cell type on days 7 and 14 following treatment compared to day 0 levels, whereas IGFBP1 was upregulated only in ESCs on days 7 and 14 compared to day 0, and not in AMCs, MMCs, and BMCs.

Decidual differentiation by treatment with cAMP. The human ESCs, AMCs, MMCs, and BMCs all showed morphological changes from a spindle-shaped appearance to large rounded cells with large round nuclei following treatment with cAMP (Fig. 4A). In addition, flow cytometry revealed that CD90, CD105, and CD166 were attenuated in the AMCs and ESCs on day 14 of the CAMP treatment (Fig. 4B and Supplementary Fig. S3) compared to day 0 levels. Together, these changes suggested that cAMP upregulates decidual differentiation in AMCs. On the other hand, CD105 and CD166 in MMCs as well as CD90 and CD166 in BMCs were unchanged, suggesting that AMCs could have more potential for differentiation to ESCs than MMCs and BMCs.

Immunofluorescence staining showed no expression of decidualization markers (PRL and IGFBP1) at day 0 in the four cell types, but positive staining for PRL on days 7 and 14 of the cAMP treatment (Fig. 5A, 5C, 5E and 5G). Although IGFBP1 was also expressed in all the types of cells on day 7 and day 14, AMCs and MMCs showed more remarkable IGFBP1 expression on day 7 compared to day 14 .

Finally, the qRT-PCR analysis showed that PRL transcripts were more abundant in all the cell types on days 7 and 14 following CAMP treatment than on day 0 (Figs. $4 \mathrm{C}$ and $4 \mathrm{D}$ ), whereas IGFBP1 was upregulated in AMCs and ESCs, but not in MMCs and BMCs. These results suggested that AMCs treated with cAMP have the closest decidualization potential to ESCs.

\section{Discussion}

Insufficient response of MSCs to $\mathbf{E}_{2}+\mathbf{P}_{4}$. In this study, we investigated whether human MSCs such as AMCs and MMCs could be differentiated in vitro into endometrial stroma-like cells using decidualization stimuli and analysis of cellular morphology and expression of decidualization markers (PRL and IGFBP1). The MSCs and ESCs were treated with $\mathrm{E}_{2}+\mathrm{P}_{4}$ to mimic the normal endocrinological condition in the secretory phase of a human menstrual cycle. ESCs and BMCs were considered as positive controls. Unexpectedly, not only the AMCs and MMCs, but also the BMCs, failed to show remarkable decidual changes with $\mathrm{E}_{2}+\mathrm{P}_{4}$ treatment. Sustained expression of cell surface markers, no marked morphological changes, and little expression of IGFBP1 in MSCs treated with $\mathrm{E}_{2}+\mathrm{P}_{4}$ indicated that these cells are not sufficiently responsive to such stimulation to induce differentiation, supporting previous results by Aghajanova. et al. ${ }^{19}$ in BMCs treated with $\mathrm{E}_{2}+\mathrm{P}_{4}$. The qRT-PCR in this study showed significant expression of progesterone receptors (PR) and estrogen receptors (ESR1 and ESR2) in AMCs, MMCs, and ESCs (Supplementary Fig. S5). Aghajanova et al. also demonstrated $P R, E S R 1$, and ESR2 mRNA expression in BMCs and ESCs, but this expression was not significantly influenced by cAMP treatment of the cells ${ }^{19}$. Furthermore, with respect to secretory-phase endometrium in vivo, decidualization of the superficial endometrial layers is only apparent $\sim 10$ days after the postovulatory increase in progesterone levels. All these observations indicate that additional signals other than the progesterone signaling are required to initiate decidualization. Interestingly, trichostatin A (TSA), a specific histone deacetylase (HDACs) inhibitor, enhanced the upregulation of PRL and IGFBP1 in a dose-dependent manner in cultured endometrial stromal cells following treatment with $\mathrm{E}_{2}+\mathrm{P}_{4}{ }^{24}$. In addition, IGFBP1 expression induced by cAMP was associated with the histone acetylation status of the promoter region in human endometrial stromal cells ${ }^{25}$. Based on these results, we speculated that TSA might be effective in enhancing IGFBP1 expression in AMCs and MMCs treated with $\mathrm{E}_{2}+\mathrm{P}_{4}$; however, AMCs and MMCs treated with $\mathrm{E}_{2}+\mathrm{P}_{4}$ and several concentrations 
A

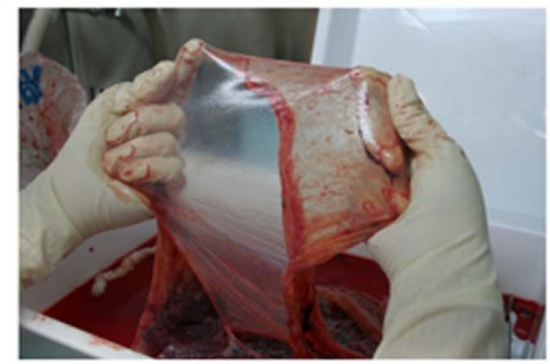

C

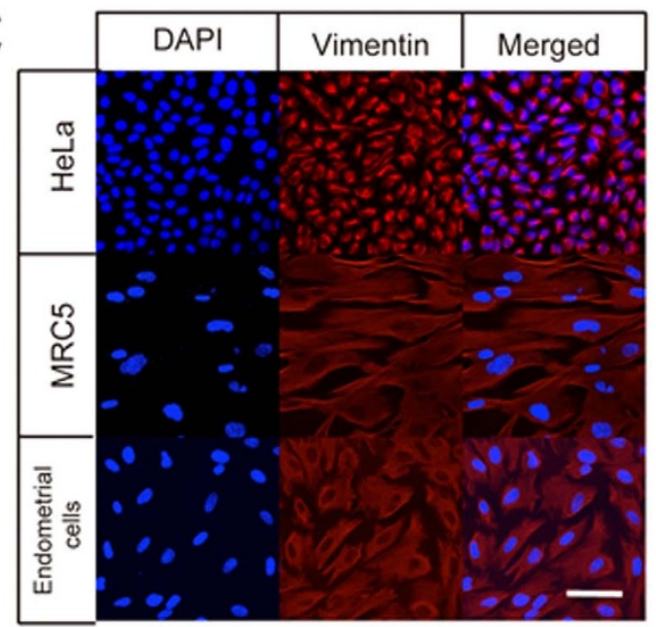

B

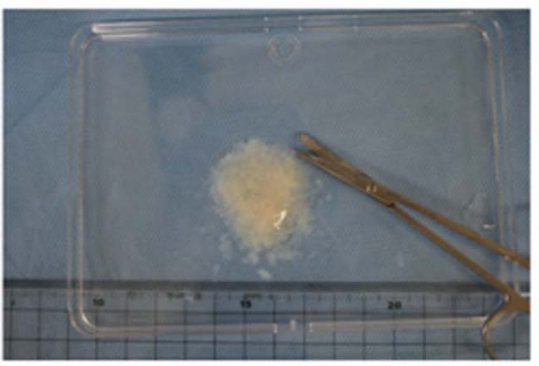

D

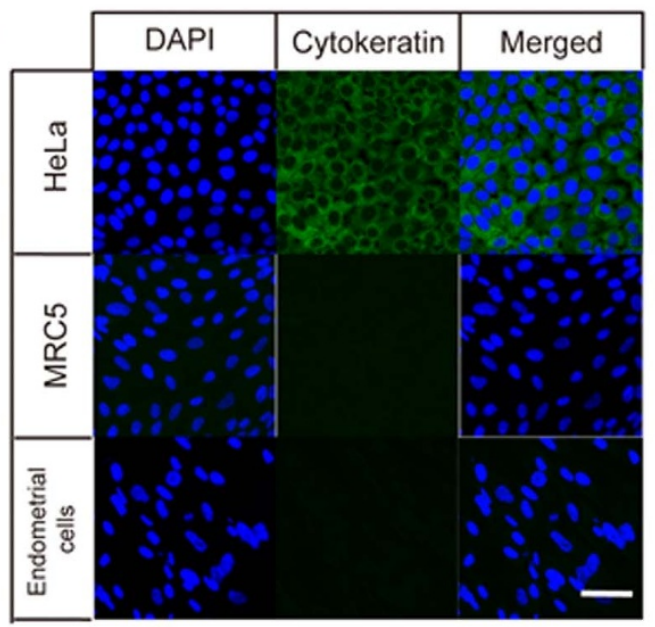

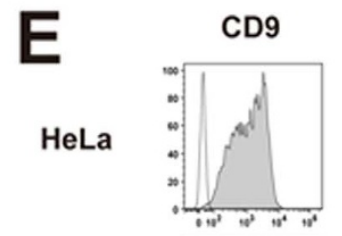
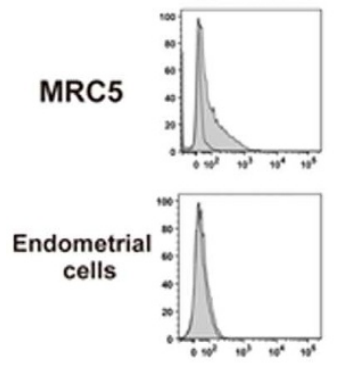
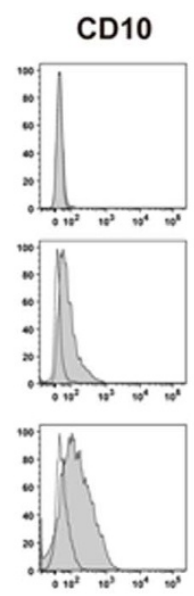

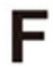

\begin{tabular}{llllllllllllll} 
Surface & $C D$ & $C D$ & $C D$ & $C D$ & $C D$ & $C D$ & $C D$ & $C D$ & $C D$ & $C D$ & $C D$ & $C D$ & $H L A$ \\
marker & 13 & 14 & 16 & 19 & 29 & 34 & 45 & 54 & 73 & 90 & 105 & 166 & -DR \\
\hline
\end{tabular}

\begin{tabular}{|c|c|c|c|c|c|c|c|c|c|c|c|c|c|}
\hline ESCs & \pm & - & - & - & \pm & - & - & + & ++ & + & + & + & - \\
\hline AMCs & \pm & - & - & - & - & - & - & + & ++ & + & + & + & - \\
\hline MMCs & \pm & - & - & - & - & - & - & + & ++ & + & + & + & - \\
\hline BMCs & \pm & - & - & - & + & - & \pm & - & ++ & ++ & + & + & - \\
\hline MRC5 & - & - & - & - & - & - & - & - & + & - & - & - & - \\
\hline
\end{tabular}

Figure 1 Characterization of endometrial cells, amnion-derived cells, and menstrual blood-derived cells. (A), Macroscopic view of human amniotic membrane. (B), The amniotic membrane was cut into pieces of approximately $2 \mathrm{~mm}^{3}$ in size. (C and D), Laser confocal microscopic view of immunofluorescence staining of endometrial cells, HeLa cells, and MRC5 fibroblasts in culture with anti-vimentin (red) (C) and anti-cytokeratin antibodies (green) (D), DAPI staining (blue). Scale bars, $100 \mu \mathrm{m}$. (E), Surface marker (CD9 or CD10) expression of endometrial cells, HeLa cells, and MRC5 fibroblasts. White and grey areas indicate reactivity of antibodies for isotype controls and that for CD9 or CD10, respectively. (F), Summary of flow cytometric analysis of endometrial stromal cells (ESCs), amnion-derived mesenchymal stem cells (AMCs), menstrual blood-derived mesenchymal stem cells (MMCs), bone marrow-derived mesenchymal stem cells (BMCs), and MRC5 fibroblasts before treatment. The shift in peak histogram value of flow cytometric data was measured and classified into four groups: -, from negative to $2 \log$ shift;, $\pm 2 \log$ shift from negative control; +, 3 log shift from negative control;,++ 4 log shift from negative control.

of TSA showed no enhancement of IGFBP1 expression (Supplemental Fig. S4). We therefore further investigated the decidualization potential of MSCs in the current study using cAMP, a well-known and common facilitator of decidualization ${ }^{19,25}$.
Attenuation of cell surface markers for MSCs during successful decidualization by cAMP. Estrogen and progesterone bind to nuclear receptor proteins for nuclear importing, where they bind DNA directly to regulate transcription, whereas cAMP activates the protein 


\section{A}
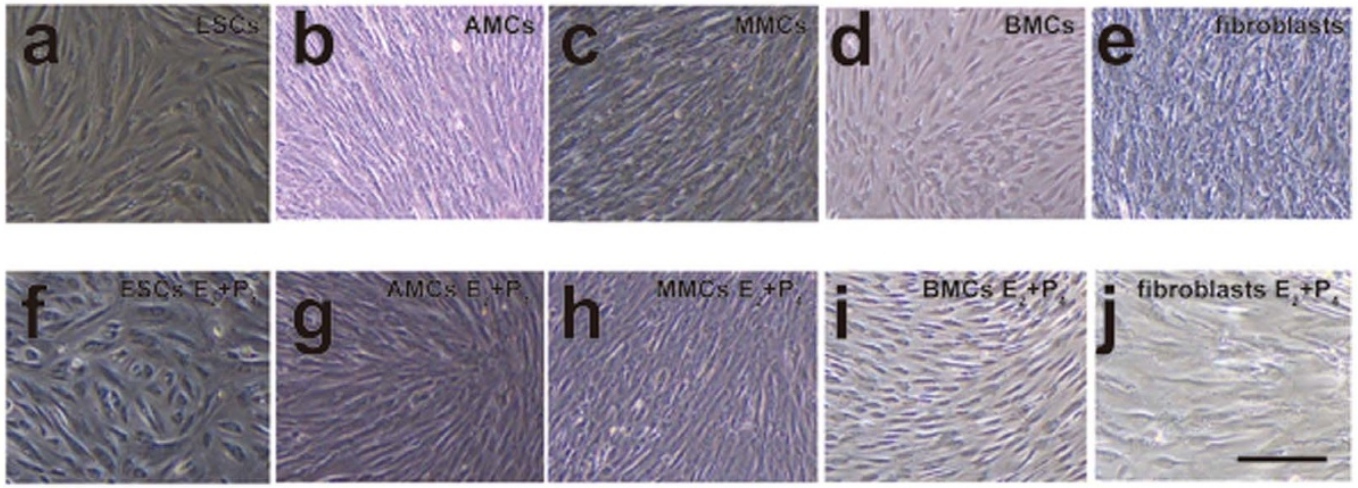

\begin{tabular}{lllllllllllllll} 
Surface & $C D$ & $C D$ & $C D$ & $C D$ & $C D$ & $C D$ & $C D$ & $C D$ & $C D$ & $C D$ & $C D$ & $C D$ & $H L A$ \\
marker & 13 & 14 & 16 & 19 & 29 & 34 & 45 & 54 & 73 & 90 & 105 & 166 & $-D R$ \\
\hline ESCs & \pm & - & - & - & + & - & - & + & + & + & + & + & - \\
AMCs & \pm & - & - & - & + & - & - & + & ++ & + & + & + & - \\
MMCs & + & - & - & - & + & - & - & + & ++ & + & + & + & - \\
BMCs & \pm & - & - & - & \pm & - & \pm & + & ++ & ++ & + & + & - \\
MRC5 & - & - & - & - & - & - & - & - & - & - & + & - & -
\end{tabular}
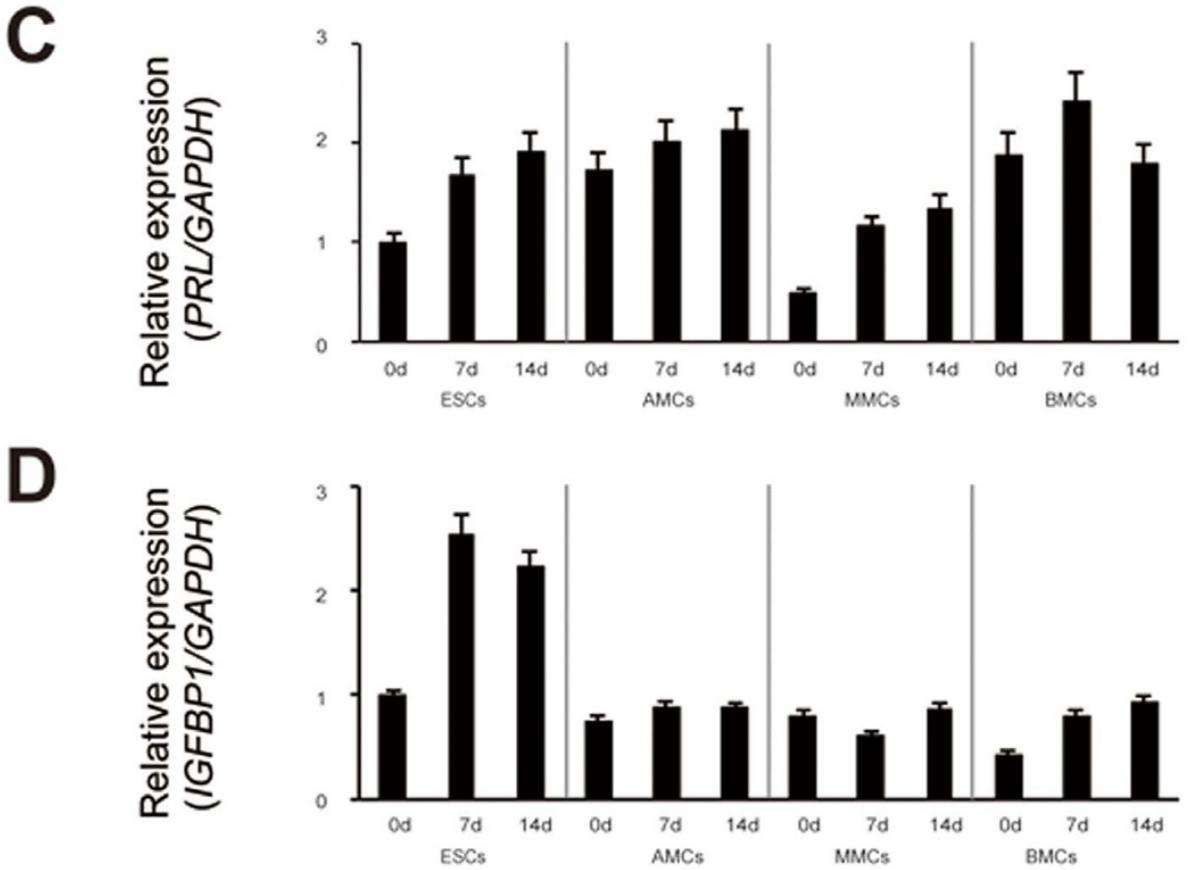

Figure $2 \mid$ Morphological changes and alteration of flow cytometric profiles in mesenchymal stem cells treated with $E_{2}+P_{4}$. (A), Morphological changes in endometrial stromal cells (ESCs) (a, f), amnion-derived mesenchymal stem cells (AMCs) (b, g), menstrual blood-derived mesenchymal stem cells (MMCs) $(c, h)$, bone marrow-derived mesenchymal stem cells (BMCs) $(d, i)$, and MRC5 fibroblasts $(e, j)$ before $(a-c)$ and after $(f-j)$ treatment with $10 \mathrm{nM} \mathrm{E}_{2}$ and $1 \mu \mathrm{M} \mathrm{P}_{4}\left(\mathrm{E}_{2}+\mathrm{P}_{4}\right)$ for 14 days. Scale bars, $200 \mu \mathrm{m}$. (B), Summary of flow cytometric analysis of ESCs, AMCs, MMCs, BMCs, and MRC5 fibroblasts treated with $\mathrm{E}_{2}+\mathrm{P}_{4}$ for 14 days. We measured the shift of peak histogram value for the flow cytometric data and defined each result by the aforementioned criteria. (C), PRL mRNA expression in ESCs, AMCs, MMCs, and BMCs treated with $\mathrm{E}_{2}+\mathrm{P}_{4}$ for 0, 7, and 14 days. (D), IGFBP1 mRNA expression in ESCs, AMCs, MMCs, and BMCs treated with $\mathrm{E}_{2}+\mathrm{P}_{4}$ for 14 days.

kinase A (PKA) pathway and a catalytic subunit of PKA translocates into the cell nucleus to phosphorylate cAMP response element-binding protein (CREB). Activated CREB then enhances gene transcription via cAMP response element $(\mathrm{CRE})^{26}$. In this study, cAMP treatment of
AMCs and MMCs produced morphological changes characteristic of decidual cells, and PRL and IGFBP1 expression increases consistent with those seen in ESCs. AMCs and MMCs were thus demonstrated to have decidualization potential. 
A ESCs

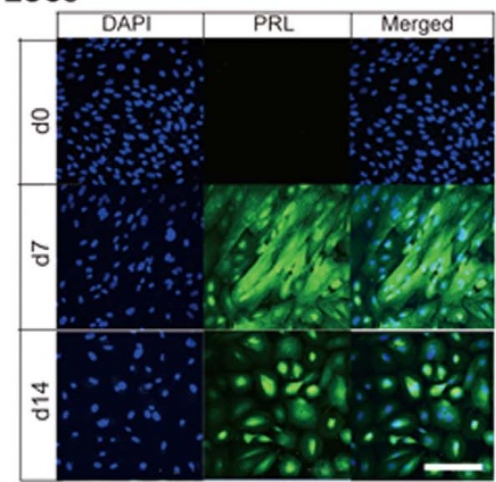

C

AMCs

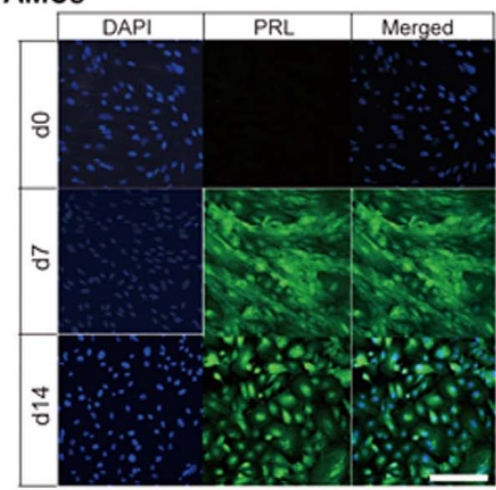

E

\section{MMCs}

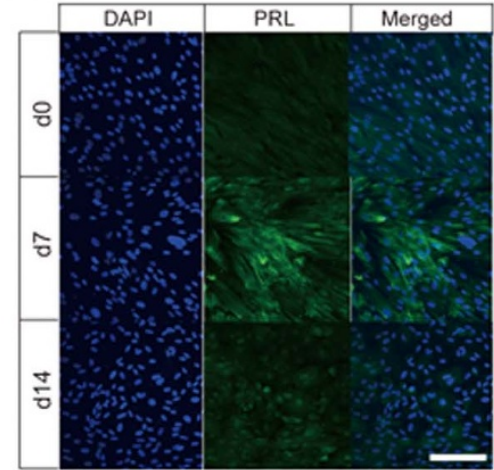

G

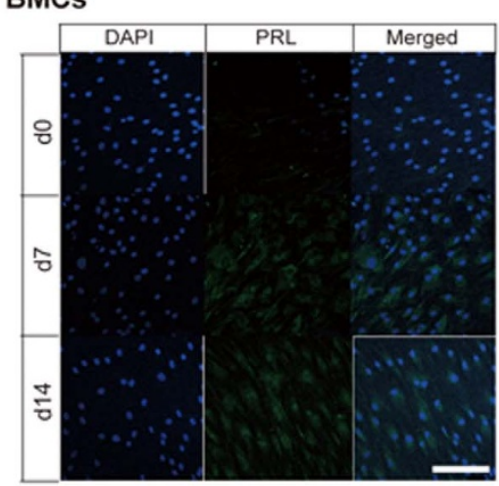

B Escs

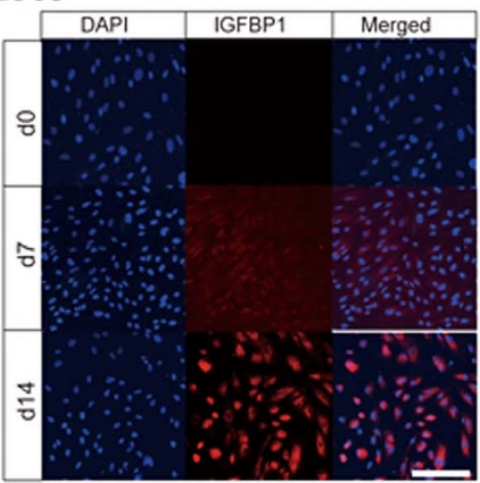

D AMCs

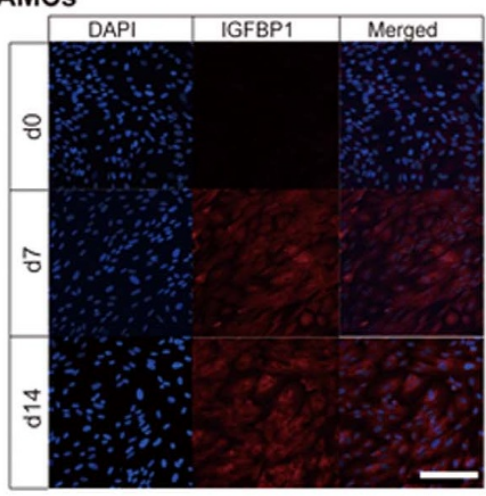

E MMCs

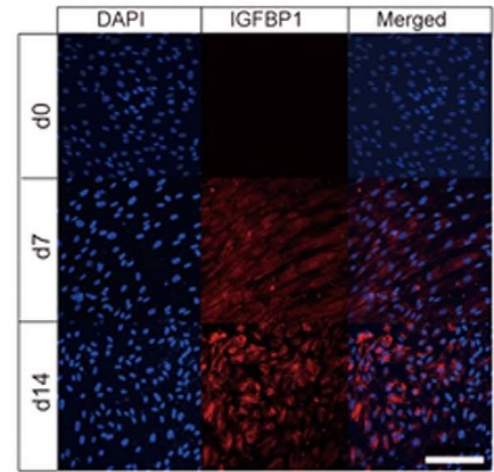

H вмсs

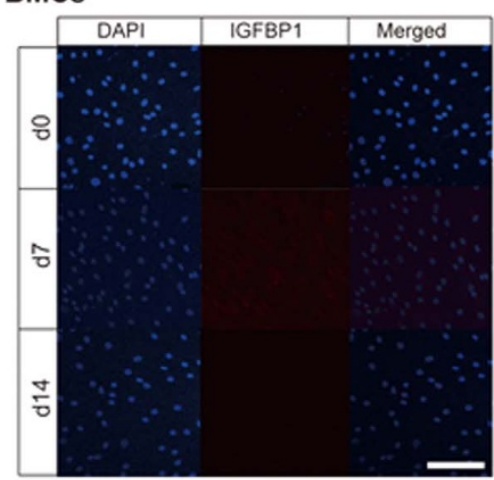

Figure $3 \mid$ Immunofluorescence staining of decidualization markers (PRL and IGFBP1) in mesenchymal stem cells treated with $\mathrm{E}_{2}+\mathrm{P}_{4}$. Laser confocal microscopy of PRL immunofluorescence staining (green signals in A, C, E, and G) and IGFBP1 (red signals in B, D, F, H) in endometrial stromal cells (ESCs) (A and B), amnion-derived mesenchymal stem cells (AMCs) (C and D), menstrual blood-derived mesenchymal stem cells (MMCs) (E and F), and bone marrow-derived mesenchymal stem cells BMCs $(\mathrm{G}$ and $\mathrm{H})$ treated with $\mathrm{E}_{2}+\mathrm{P}_{4}$ for 0 , 7, or 14 days. Scale bars, $200 \mu \mathrm{m}$. 


\section{A}
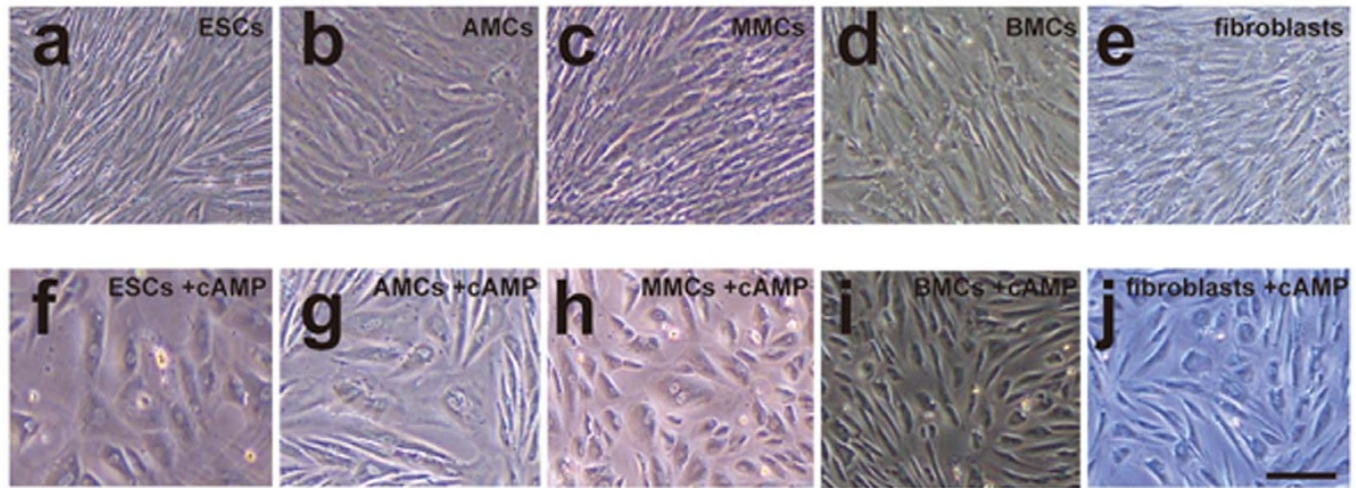

\begin{tabular}{lllllllllllllllll} 
Burface & $C D$ & $C D$ & $C D$ & $C D$ & $C D$ & $C D$ & $C D$ & $C D$ & $C D$ & $C D$ & $C D$ & $C D$ & $H L A$ \\
marker & 13 & 14 & 16 & 19 & 29 & 34 & 45 & 54 & 73 & 90 & 105 & 166 & $-D R$ \\
\hline ESCs & - & - & - & - & - & - & - & + & ++ & - & \pm & \pm & - \\
AMCs & \pm & - & - & - & - & - & - & \pm & ++ & \pm & \pm & \pm & - \\
MMCs & + & \pm & \pm & \pm & \pm & \pm & \pm & ++ & ++ & \pm & + & + & \pm \\
BMCs & \pm & \pm & - & - & \pm & - & - & + & + & ++ & \pm & + & - \\
MRC5 & - & - & - & - & - & - & - & - & - & - & - & - & - \\
\hline
\end{tabular}
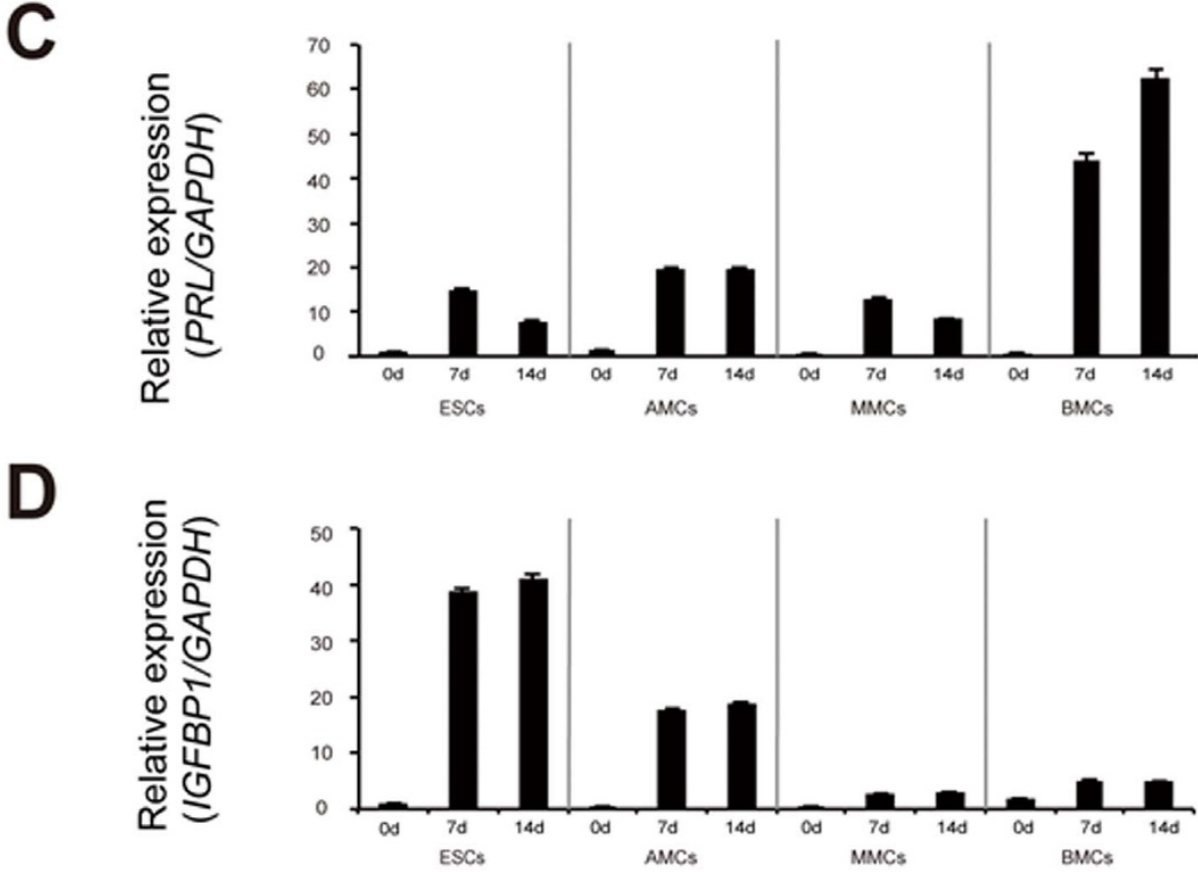

Figure $4 \mid$ Morphological changes and alteration of flow cytometric profiles in mesenchymal stem cells treated with cAMP. (A), Morphological changes of endometrial stromal cells (ESCs) (a, f), amnion-derived mesenchymal stem cells (AMCs) (b, g), menstrual blood-derived mesenchymal stem cells (MMCs) (c, h), bone marrow-derived mesenchymal stem cells (BMCs) $(\mathrm{d}, \mathrm{i})$, and MRC5 fibroblasts $(\mathrm{e}, \mathrm{j})$ before $(\mathrm{a}-\mathrm{e})$ and $(\mathrm{f}-\mathrm{j})$ after treatment with $1 \mathrm{mM} 8$-Br-cAMP for 14 days. Scale bars, $200 \mu \mathrm{m}$. (B), Summary of flow cytometric analysis of ESCs, AMCs, MMCs, BMCs, and MRC5 fibroblasts treated with $1 \mathrm{mM} 8$-Br-cAMP for 14 days. We measured the shift of peak histogram value for the flow cytometric data and defined each result by the aforementioned criteria. (C), PRL mRNA expression in ESCs, AMCs, MMCs, and BMCs treated with 1 mM 8-Br-cAMP for 0, 7, and 14 days. (D), IGFBP1 mRNA expression in ESCs, AMCs, MMCs, and BMCs treated with $1 \mathrm{mM} \mathrm{8-Br-cAMP}$ for 0, 7, and 14 days.

In the cell surface marker analysis, the attenuation of CD90, CD105, and CD166 during decidualization was notable. Such a response by these well-defined human MSCs markers could indicate the direction of differentiation in MSCs treated with cAMP.
Furthermore, CD90 is known to regulate adhesion to T cells in MSCs, and a decrease in CD90-positive cells might be a novel predictive marker of immunosuppressive activity ${ }^{27}$. Based on these observations, the decreased rate of CD90-positive cells with cAMP 

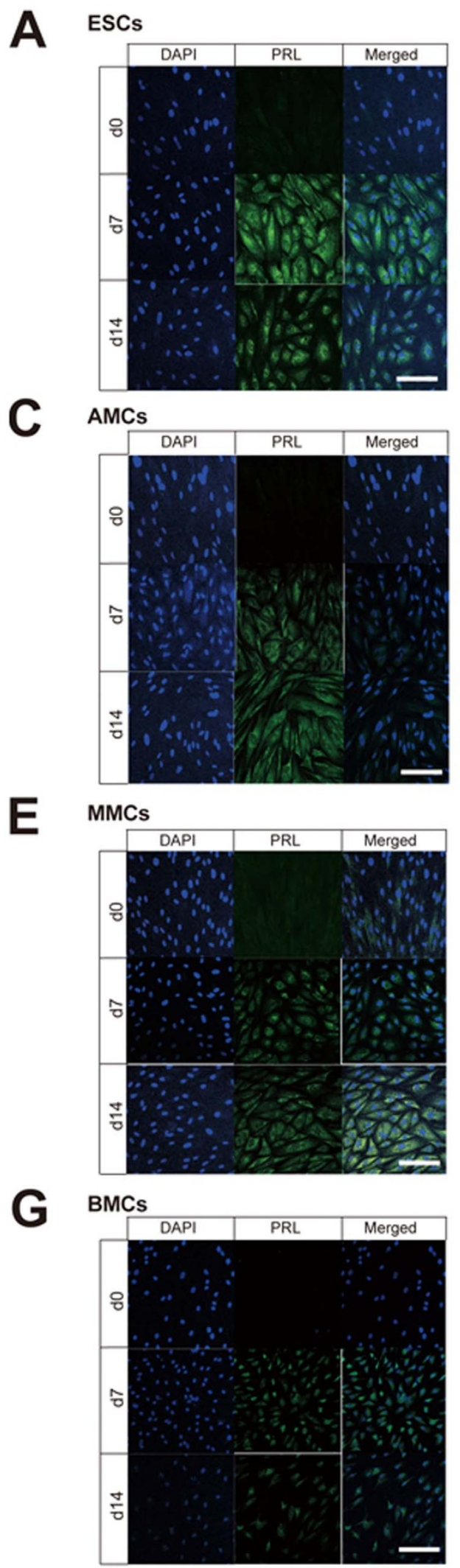

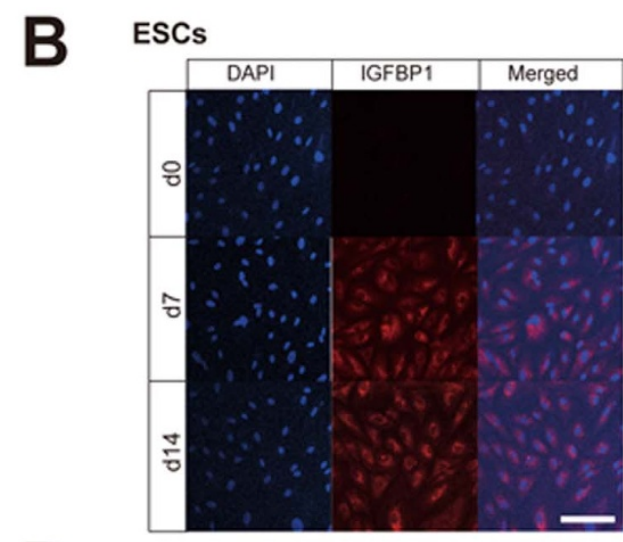

D AMCs

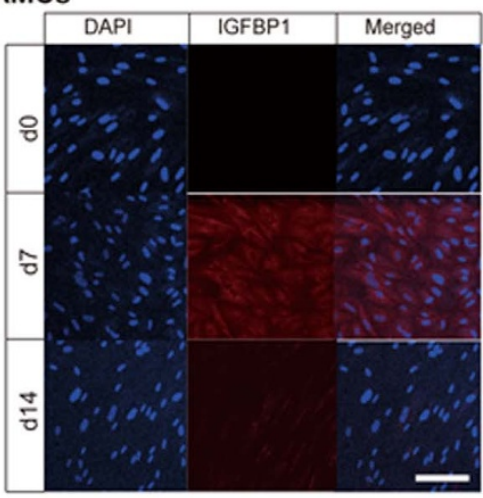

F $\quad$ MMCs

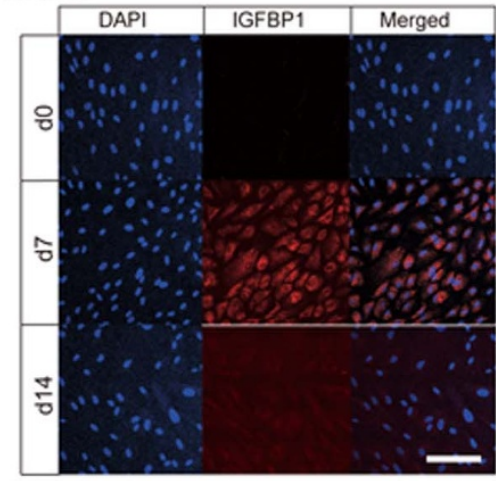

H

BMCs

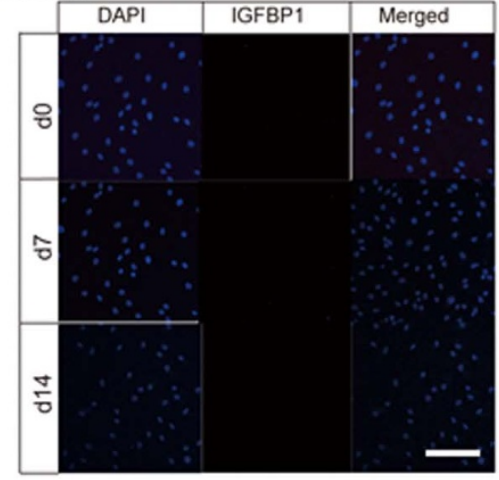

Figure 5 Immunofluorescence staining of decidualization markers (PRL and IGFBP1) in mesenchymal stem cells treated with cAMP. Laser confocal microscopic view of immunofluorescence staining for PRL (green signals in A, C, E, and G) and IGFBP1 (red signals in B, D, F, and H) in endometrial stromal cells (ESCs) (A and B), amnion-derived mesenchymal stem cells (AMCs) (C and D), menstrual blood-derived mesenchymal stem cells (MMCs) (E and F), bone marrow-derived mesenchymal stem cells (BMCs) (G and H) treated with $1 \mathrm{mM} \mathrm{8-Br-cAMP}$ for 0, 7, and 14 days. Scale bars, $200 \mu \mathrm{m}$. 
observed in the current study may indicate immunosuppression in AMCs and MMCs that is advantageous in terms of their potential clinical application. CD105 (endoglin) is a reliable marker for highly multipotent human MSCs, with CD105-expressing MSCs showing high efficiency in repairing the infarcted heart ${ }^{28}$. Therefore, the disappearance of CD105 with cAMP treatment would ensure differentiation of $\mathrm{MSCs}^{29,30}$. Collectively, our findings indicated that administration of cAMP effectively leads to differentiation and decidualization of AMCs and MMCs.

Advantages of AMCs and MMCs. It has been reported that endometrial stem cells are CD45-positive cells around blood vessels and likely to be derived from $\mathrm{BMCs}^{31}$. A previous study also indicated that BMCs might be a source of endometrial stem/ progenitor cells based on successful decidualization and gene expression profiles of $\mathrm{BMCs}$ treated with $\mathrm{CAMP}^{19}$. If $\mathrm{BMCs}$ are indeed a physiological source of endometrial progenitor cells, BMCs could be ideal for use in cell-based therapies for infertile women who have thinning of the endometrium. However, our findings revealed that not only BMCs, but AMCs and MMCs, also showed decidualization potential, and that all three cell types showed a similar profile of cell surface marker expression to that of ESCs. Therefore, the current study suggested that BMCs is not the only such candidate for cell therapy.

In general, the collection of bone marrow is an invasive technique performed with a biopsy needle that requires local or even general anesthesia, and occasionally raises an ethical problem. On the other hand, human amnion is non-invasively and adequately obtained at delivery. Therefore, human amnion is more simply accessed with informed consent than other potential cell sources. Human primary menstrual blood-derived cells are also obtained by a simple, safe, and painless procedure, and efficiently expanded in vitro. In particular, the MMC-derived cells can be used for autologous transplantation. Accordingly, if culture condition for MMCs could be more successfully optimized for differentiation to ESCs, MMCs would be definitely useful for eventual cell-based therapies for infertility.

Furthermore, previous studies suggested that human amnion has the potential for immunological tolerance ${ }^{23,32}$. AMCs are known to express less MHC compared to other MSCs, which is important in host tolerance. Cells that do not express MHC are potentially recognized by the immune system as non-self cells and accordingly, attacked by natural killer (NK) cells. A soluble factor such as the non-classic MHC class I antigen, HLA-G, is also critical for the immunosuppressive properties of MSCs ${ }^{33}$. HLA-G is expressed on the extravillous cytotrophoblast cells at the fetomaternal interface and plays a major role in protecting the fetus from maternal rejection by NK cells ${ }^{34,35}$. HLA-G thus blocks the immunological response of $\mathrm{NK}$ cells ${ }^{36}$ and induces regulatory $\mathrm{T}_{\text {cells }}{ }^{37}$, which is a requisite condition of immunological tolerance ${ }^{35,38}$. We previously showed by western blotting that HLA-G is also expressed in placenta-derived tissues including amnion ${ }^{23}$. Thus, AMC-derived cells have several advantages for application to cell-based therapy.

Based on the results of our current study, AMCs and MMCs in addition to BMCs show potential for decidualization, and are therefore candidate cell sources for therapy to support implantation and placentation. The current study provides novel and important information on AMCs and MMCs treated with cAMP as a model of decidualization.

\section{Methods}

Tissue isolation and cell culture. All experiments involving human cells and tissues were performed according to the Tenets of the Declaration of Helsinki and were approved by the Ethics Committee of the National Institute for Child and Health Development, Tokyo. Signed informed consent was obtained from all donors, and all specimens were irreversibly de-identified. Human endometrium was obtained from a patient undergoing hysterectomy due to myoma uteri and human placenta was collected after selective caesarean section delivery.
Human amnion-derived cells were isolated using the explant culture method, in which the cells were outgrown from pieces of amnion. Briefly, the amnion was cut into pieces of approximately $2 \mathrm{~mm}^{3}$ in size, which were then washed in Dulbecco's modified Eagle's medium (DMEM) (high glucose; Sigma) supplemented with 100 $\mathrm{U} / \mathrm{ml}$ penicillin-streptomycin (Gibco). Some pieces were attached to the substratum in a $10-\mathrm{cm}$ dish. The cells migrated out from the cut ends after approximately 20 days of incubation at $37^{\circ} \mathrm{C}$ in $5 \% \mathrm{CO}_{2}$. The migrated cells were harvested with Dulbecco's phosphate-buffered saline containing $0.1 \%$ trypsin and $0.25 \mathrm{mM}$ EDTA for $5 \mathrm{~min}$ at $37^{\circ} \mathrm{C}$ and counted. The harvested cells were then re-seeded at a density of $3 \times 10^{5}$ cells in 10-cm dishes. Once confluent, the cell monolayers were subcultured at a $1: 8 \mathrm{split}$ ratio onto new $10-\mathrm{cm}$ dishes, and the culture medium was replaced with fresh DMEM supplemented with $10 \%$ fetal bovine serum (FBS) and $100 \mathrm{U} / \mathrm{ml}$ penicillin-streptomycin every 3 or 4 days $^{22}$.

Menstrual blood samples $(n=21)$ were collected in DMEM containing $100 \mathrm{U} / \mathrm{ml}$ penicillin-streptomycin and $2 \% \mathrm{FBS}$, and processed within $24 \mathrm{~h}$. The centrifuged pellets containing endometrium-derived cells were resuspended in high-glucose DMEM with $10 \%$ FBS and penicillin-streptomycin, and then maintained at $37^{\circ} \mathrm{C}$ in a humidified atmosphere containing $5 \% \mathrm{CO}_{2}$ to attach for $48 \mathrm{~h}$. Non-adherent cells were removed by changing the medium. Once sub-confluent, the cells were harvested with $0.25 \%$ trypsin and $1 \mathrm{mM}$ EDTA, and plated to new dishes. After $2-3$ passages, the attached cells were devoid of blood cells ${ }^{21}$.

A human bone marrow-derived mesenchymal cell line, 3F0664 was purchased from Lonza (PT-2501, Basel, Switzerland) and cultured in the mesenchymal stemcell-basal-medium (MSCBM)-Medium-BulletKit (PT-3238, Lonza). The normal human fetal fibroblast cell line MRC5 was also passaged in DMEM supplemented with $10 \%(\mathrm{v} / \mathrm{v}) \mathrm{FBS}$ and $100 \mathrm{U} / \mathrm{ml}$ penicillin-streptomycin. Cultures were maintained at $37^{\circ} \mathrm{C}$ with $5 \% \mathrm{CO}_{2}$ in a humidified atmosphere.

For decidual differentiation, human endometrial cells, amnion-derived cells, menstrual blood-derived cells, bone marrow-derived cells, and MRC5 were first cultured as described above, then trypsinized and plated in $10-\mathrm{cm}$ plates. Nearly confluent cells were cultured for 0,7 , and 14 days in low-serum medium (2\% FBS) supplemented with: 1) $10 \mathrm{nM}$ estrogen $\left(\mathrm{E}_{2}\right)$ and $1 \mu \mathrm{M}$ progesterone $\left(\mathrm{P}_{4}\right)$ or 2$) 1 \mathrm{mM}$ 8-Br-cAMP (cAMP) (Sigma-Aldrich, Saint Louis, MO, USA).

Flow cytometric analysis. Cells were incubated for $20 \mathrm{~min}$ at $4{ }^{\circ} \mathrm{C}$ with primary antibodies or isotype-matched control antibodies, followed by immunofluorescent secondary antibodies. The cells were then analyzed on a FACS Aria ${ }^{\mathrm{TM}}$ IIIu Cellsorter (Becton Dickinson, Inc.) using FlowJo Ver.7 (Tree Star, Inc.). Antibodies against human CD9, CD10, CD13, CD14, CD16, CD19, CD29, CD31, CD34, CD44, CD45, CD54, CD55, CD59, CD73, CD90, CD105, CD133, CD146, CD166, HLA-ABC, and HLA-DR were purchased from Beckman Coulter, Immunotech (Marseille, France), BD Pharmingen and BioLegend (San Diego, CA, USA) ${ }^{21,22}$. Supplementary table S1 shows the list of CD antigens investigated in this study ${ }^{39-49}$.

Immunofluorescence staining. The immunofluorescence staining was performed as previously described ${ }^{50}$. Briefly, cells were fixed with $4 \%$ paraformaldehyde for $10 \mathrm{~min}$ at $4{ }^{\circ} \mathrm{C}$, treated with $0.1 \%$ Triton X-100 (Sigma) in PBS for $15 \mathrm{~min}$ at RT, and then incubated for $30 \mathrm{~min}$ at RT in protein-blocking solution consisting of PBS supplemented with 5\% FBS. The samples were then incubated overnight with the following primary antibodies diluted in PBS: an anti-prolactin mouse monoclonal antibody, (QED Bioscience, San Diego, CA, USA), anti-IGFBP1 mouse monoclonal antibody (Santa Cruz Biotechnology, Dallas, Texas, CA, USA), anti-vimentin mouse monoclonal antibody, V9, (DAKO, Tokyo, Japan), and an anti-cytokeratin mouse monoclonal antibody, AE1 + AE3, (DAKO). Tissues were washed twice for $5 \mathrm{~min}$ in PBS and incubated with the appropriate secondary antibodies (Alexa Fluor 488 goat anti-mouse IgG, 1:600 and Alexa Fluor 546 goat anti-mouse IgG, 1:600; Life Technologies, Grand Island, NY). Nuclei were counterstained with 4', 6-diamidino2-phenylindole (DAPI; Biotium, CA, USA) for $30 \mathrm{~min}$ at RT followed by 10 final 5 min PBS washes before being cover slipped using mounting medium. Omission of primary antibodies served as a negative control.

Quantitative reverse transcriptase-polymerase chain reaction (qRT- PCR) analysis. Total cellular RNA was isolated from cells using an RNeasy Plus Mini Kit (Qiagen, Hilden, Germany) according to the manufacturer's protocol. Total RNA (2.5 $\mu \mathrm{g}$ each) for quantitative RT-PCR was converted to cDNA using an Oligo (dT) primer with Superscript VILO ${ }^{\mathrm{TM}}$ cDNA Synthesis Kit (Life Technologies Corp., Carlsbad, CA, USA), according to the manufacturer's manual. The cDNA template was amplified by thermal cycle reactions (Quant Studio ${ }^{\mathrm{TM}} 12 \mathrm{~K}$ Flex Real-Time PCR System) using the Platinum SYBR Green qPCR SuperMix-UDG (Life Technologies Corp.) under the following reaction conditions: 40 cycles of PCR $\left(95^{\circ} \mathrm{C}\right.$ for $15 \mathrm{~s}$ and $60^{\circ} \mathrm{C}$ for $\left.1 \mathrm{~min}\right)$ after an initial denaturation $\left(95^{\circ} \mathrm{C}\right.$ for $\left.2 \mathrm{~min}\right)$. Fluorescence was monitored during every PCR cycle at the annealing step. The authenticity and size of the PCR products were confirmed by a melting curve analysis using software provided by Applied Biosystems. mRNA levels were normalized using GAPDH as a housekeeping gene. Primers used to amplify a cDNA fragment for human PRL and IGFBP1 were the following: PRL, 5' -CATCAACAGCTGCCACACTT- $3^{\prime}$ (F) and 5' -CGTTTGGTTTGCTCCTCAAT-3' (R); IGFBP1, 5' -CTATGATGGCTCGAAGGCTC-3' (F) and 5' -TTCTTGTTGCAGTTTGGCAG-3' (R).

1. Lee, T. H. et al. Embryo quality is more important for younger women whereas age is more important for older women with regard to in vitro fertilization outcome and multiple pregnancy. Fertil Steril 86, 64-69 (2006). 
2. Navot, D. et al. Poor oocyte quality rather than implantation failure as a cause of age-related decline in female fertility. Lancet 337, 1375-1377 (1991).

3. Amir, W. et al. Predicting factors for endometrial thickness during treatment with assisted reproductive technology. Fertil Steril 87, 799-804 (2007).

4. Senturk, L. M. \& Erel, C. T. Thin endometrium in assisted reproductive technology. Curr Opin Obstet Gynecol 20, 221-228 (2008).

5. Sallam, H. N. Embryo transfer: factors involved in optimizing the success. Curr Opin Obstet Gynecol 17, 289-298 (2005).

6. Check, J. H. et al. Neither sildenafil nor vaginal estradiol improves endometrial thickness in women with thin endometria after taking oral estradiol in graduating dosages. Clin Exp Obstet Gynecol 31, 99-102 (2004).

7. Landgren, B. M., Johannisson, E., Stavreus-Evers, A., Hamberger, L. \& Eriksson, H. A new method to study the process of implantation of a human blastocyst in vitro. Fertil Steril 65, 1067-1070 (1996)

8. Eyheremendy, V. et al. Beneficial effect of autologous endometrial cell coculture in patients with repeated implantation failure. Fertil Steril 93, 769-773 (2010).

9. Lee, E. J. et al. Spherical bullet formation via E-cadherin promotes therapeutic potency of mesenchymal stem cells derived from human umbilical cord blood for myocardial infarction. Mol Ther 20, 1424-1433 (2012).

10. Qiu, Y., Marquez-Curtis, L. \& Janowska-Wieczorek, A. Mesenchymal stromal cells derived from umbilical cord blood migrate in response to complement C1q. Cytotherapy 14, 285-295 (2012).

11. Moorefield, E. C. et al. Cloned, CD117 selected human amniotic fluid stem cells are capable of modulating the immune response. PLoS One 6, e26535 (2011)

12. Gargett, C. E. \& Healy, D. L. Generating receptive endometrium in Asherman's syndrome. J Hum Reprod Sci 4, 49-52 (2011).

13. Pittenger, M. F. et al. Multilineage Potential of Adult Human Mesenchymal Stem Cells. Science 284, 143-147 (1999).

14. Miki, T., Lehmann, T., Cai, H., Stolz, D. B. \& Strom, S. C. Stem cell characteristics of amniotic epithelial cells. Stem Cells 23, 1549-1559 (2005).

15. Santos, T. M. et al. Expression of pancreatic endocrine markers by mesenchymal stem cells from human umbilical cord vein. Transplant Proc 42, 563-565 (2010).

16. Lee, K. D. et al. In vitro hepatic differentiation of human mesenchymal stem cells. Hepatology 40, 1275-1284 (2004).

17. Pournasr, B. et al. In vitro differentiation of human bone marrow mesenchymal stem cells into hepatocyte-like cells. Arc Iran Med 14, 244-249 (2011)

18. Dezawa, M. et al. Specific induction of neuronal cells from bone marrow stromal cells and application for autologous transplantation. J Clin Invest 113, 1701-1710 (2004)

19. Aghajanova, L., Horcajadas, J. A., Esteban, F. J. \& Giudice, L. C. The bone marrowderived human mesenchymal stem cell: potential progenitor of the endometrial stromal fibroblast. Biol Reprod 82, 1076-1087 (2010).

20. Diaz-Prado, S. et al. Human amniotic membrane as an alternative source of stem cells for regenerative medicine. Differentiation 81, 162-171 (2011).

21. Cui, C. H. et al. Menstrual blood-derived cells confer human dystrophin expression in the murine model of Duchenne muscular dystrophy via cell fusion and myogenic transdifferentiation. Mol Biol Cell 18, 1586-1594 (2007).

22. Kawamichi, Y. et al. Cells of extraembryonic mesodermal origin confer human dystrophin in the mdx model of Duchenne muscular dystrophy. J Cell Physiol 223 695-702 (2010).

23. Tsuji, H. et al. Xenografted human amniotic membrane-derived mesenchymal stem cells are immunologically tolerated and transdifferentiated into cardiomyocytes. Circ Res 106, 1613-1623 (2010).

24. Sakai, N. et al. Involvement of histone acetylation in ovarian steroid-induced decidualization of human endometrial stromal cells. J Biol Chem $\mathbf{2 7 8}$, 16675-16682 (2003)

25. Tamura, I. et al. Induction of IGFBP-1 expression by cAMP is associated with histone acetylation status of the promoter region in human endometrial stromal cells. Endocrinology 153, 5612-5621 (2012).

26. Malumbres, M. \& Pellicer, A. RAS pathways to cell cycle control and cell transformation. Front Biosci 3, d887-912 (1998).

27. Campioni, D. et al. A decreased positivity for CD90 on human mesenchyma stromal cells (MSCs) is associated with a loss of immunosuppressive activity by MSCs. Cytometry B Clin Cytom 76, 225-230 (2009).

28. Gaebel, R. et al. Cell origin of human mesenchymal stem cells determines a different healing performance in cardiac regeneration. PLoS One 6, e15652 (2011).

29. Rosu-Myles, M., Fair, J., Pearce, N. \& Mehic, J. Non-multipotent stroma inhibit the proliferation and differentiation of mesenchymal stromal cells in vitro. Cytotherapy 12, 818-830 (2010).

30. Jin, H. J. et al. Down-regulation of CD105 is associated with multi-lineage differentiation in human umbilical cord blood-derived mesenchymal stem cells. Biochem Biophys Res Commun 381, 676-681 (2009).

31. Bratincsák, A. et al. CD45-positive blood cells give rise to uterine epithelial cells in mice. Stem Cells 25, 2820-2826 (2007).

32. Cui, C. H. et al. Dystrophin conferral using human endothelium expressing HLA$\mathrm{E}$ in the non-immunosuppressive murine model of Duchenne muscular dystrophy. Hum Mol Genet 20, 235-244 (2011).

33. Selmani, Z. et al. HLA-G is a crucial immunosuppressive molecule secreted by adult human mesenchymal stem cells. Transplantation 87, S62-S66 (2009).
34. Rouas-Freiss, N., Goncalves, R. M., Menier, C., Dausset, J. \& Carosella, E. D. Direct evidence to support the role of HLA-G in protecting the fetus from maternal uterine natural killer cytolysis. Proc Natl Acad Sci USA 94, 11520-11525 (1997).

35. Fernandez, N. et al. A critical review of the role of the major histocompatibility complex in fertilization, preimplantation development and feto-maternal interactions. Hum Reprod Update 5, 234-248 (1999).

36. Pazmany, L. et al. Protection from natural killer cell-mediated lysis by HLA-G expression on target cells. Science 274, 792-795 (1996).

37. Selmani, Z. et al. Human leukocyte antigen-G5 secretion by human mesenchymal stem cells is required to suppress $\mathrm{T}$ lymphocyte and natural killer function and to induce CD4 + CD25highFOXP3 + regulatory T cells. Stem Cells 26, 212-222 (2008).

38. Hutter, H. et al. Expression of HLA class I molecules in human first trimester and term placenta trophoblast. Cell Tissue Res 286, 439-447 (1996).

39. Paietta, E. Expression of cell-surface antigens in acute promyelocytic leukaemia. Best Practice \& Research Clinical Haematology 16, 369-385 (2003).

40. Gayoso, I. et al. Immunosenescence of human natural killer cells. J Innate Immun 3, 337-343 (2011).

41. Fujimoto, M. \& Sato, S. B cell signaling and autoimmune diseases: CD19/CD22 loop as a B cell signaling device to regulate the balance of autoimmunity. J Dermatol Sci 46, 1-9 (2007).

42. Mafi, P., Hindocha, S., Mafi, R., Griffin, M. \& Khan, W. S. Adult mesenchymal stem cells and cell surface characterization - a systematic review of the literature The Open Orthopaedics Journal 5, 253-260 (2011).

43. Han, K. et al. Human amnion-derived mesenchymal stem cells are a potential source for uterine stem cell therapy. Cell Prolif 41, 709-725 (2008).

44. Nery, A. A. et al. Human mesenchymal stem cells: from immunophenotyping by flow cytometry to clinical applications. Cytometry Part A 83, 48-61 (2013).

45. Kasprzak, A., Surdacka, A., Tomczak, M. \& Konkol, M. Role of high endothelial postcapillary venules and selected adhesion molecules in periodontal diseases: a review. J Periodontal Res 48, 1-21 (2013).

46. Elghetany, M. T. \& Lacombe, F. Physiologic variations in granulocytic surface antigen expression: impact of age, gender, pregnancy, race, and stress. J Leukoc Biol 75, 157-162 (2004)

47. Crisan, M., Corselli, M., Chen, W. C. W. \& Péault, B. Perivascular cells for regenerative medicine. J Cell Mol Med 16, 2851-2860 (2012).

48. Alakel, N. et al. Direct contact with mesenchymal stromal cells affects migratory behavior and gene expression profile of CD133 + hematopoietic stem cells during ex vivo expansion. Exp Hematol 37, 504-513 (2009).

49. Dvorakova, J., Hruba, A., Velebny, V. \& Kubala, L. Isolation and characterization of mesenchymal stem cell population entrapped in bone marrow collection sets. Cell Biol Int 32, 1116-1125 (2008).

50. Mori, T. et al. Combination of hTERT and bmi-1, E6, or E7 induces prolongation of the life span of bone marrow stromal cells from an elderly donor without affecting their neurogenic potential. Mol Cell Biol 25, 5183-5195 (2005).

\section{Acknowledgments}

We express our sincere thanks to Hatsune Makino, Kazumi Takanashi, Masanori Ihara, Tohru Sugawara, Yuichiro Harada, Yayoi Kajiwara, Tetsuo Maruyama, Hiroshi Uchida, Hirotaka Masuda, and other members of the National Research Institute for Child Health and Development and Keio University School of Medicine for valuable discussions and helpful advice. We also thank Kayoko Saito for secretarial work. This work was supported, in part, by a National Grant-in-Aid from the Japanese Ministry of Health, Labor, and Welfare (NCCHD24-6 to T.H. and H.A.), a Grant-in-Aid from the Japan Health Sciences Foundation (KHD1220 to T.H. and H.A.), and Grants-in-Aid from the Japan Society for the Promotion of Science (Kiban-C-23592413 to T.H. and Wakate-B-25861518 to K.S.)

\section{Author contributions}

K.S. and T.H. contributed to the experimental design, data acquisition, analysis and interpretation, and drafting of the article. M.Y., S.K. and S.O. provided technical support and assisted with the data analysis and interpretation. N.K., H.A., K.M., Y.Y. and A.U. assisted with experimental design as well as data analysis and interpretation. All authors examined the data and approved the final manuscript.

\section{Additional information}

Supplementary information accompanies this paper at http://www.nature.com/ scientificreports

Competing financial interests: The authors declare no competing financial interests. How to cite this article: Sugawara, K. et al. Derivation of human decidua-like cells from amnion and menstrual blood. Sci. Rep. 4, 4599; DOI:10.1038/srep04599 (2014).

This work is licensed under a Creative Commons Attribution-NonCommercialNoDerivs 3.0 Unported License. The images in this article are included in the article's Creative Commons license, unless indicated otherwise in the image credit; if the image is not included under the Creative Commons license, users will need to obtain permission from the license holder in order to reproduce the image. To view a copy of this license, visit http://creativecommons.org/licenses/by-nc-nd/3.0/ 